\title{
Total diz artroplastisinde çimentolu veya çimentosuz uygulamalar arası başarı kıyaslaması
}

\author{
Success comparison between cemented and cementless fixation \\ in total knee arthroplasty
}

\author{
Ömer Faruk Bilgen, Osman Yaray
}

Özel Medicabil Hastanesi, Ortopedi ve Travmatoloji Kliniği, Bursa

\begin{abstract}
İlerlemiş diz artrozunda total diz protezi, en etkili, güvenilir, sonuçları tahmin edilebilir tedavi yöntemlerinden biridir. Tüm dünyada yaygın olarak çimentolu diz protezi yapılmakta ve diz osteoartriti tedavisinde altın standart olarak kabul edilmektedir. Son zamanlarda gelişen biyoteknoloji ile birlikte, çimentosuz diz protezlerine olan ilgi artmıştır. Çimentosuz diz protezlerinin çimentolu proteze göre en büyük avantajları; biyolojik tespit yapılması, kemik stoğunun korunması, çimento debrisinden korunma ve ameliyat süresinin kısalmasıdır. Diz artroplastisi yapılan hasta popülasyonunda giderek ortalama yaşın azaldığı göz önüne alındığında, diz protezinde sağkalım süresinin uzun olması gittikçe daha önemli kriterlerden biri haline gelmiştir. Yapılan karşılaştırmalı çalışmalara ve meta-analizlere göre, çimentosuz diz protezlerinde, kısa dönemde çimentolu protez ile kıyaslanabilir düzeyde sağkalım elde edilmiş, genç ve obez hastalarda çimentosuz protez önerilmiştir. Ancak, total diz protezinde ideal tespit yöntemi hala tartışılmakta olup, cerrahların birçoğu çimentolu total diz protezini tercih etmektedir.
\end{abstract}

Anahtar sözcükler: primer total diz protezi; çimentolu; çimentosuz; klinik sonuç; aseptik gevşeme
Total knee replacement is an affective, predictable, reliable treatment for advanced knee osteoarthritis. The most common and widespread method is cemented arthroplasty, and it is accepted as golden standard for treatment of osteoarthritis of knee. With recent developments of biotechnologies, uncemented total knee arthroplasty is becoming an attractive option. The most important advantages of uncemented total knee arthroplasty are: bone stock preservation, obtaining biological fixation, cement debris protection, and shorter operation time. As the mean age of patients undergoing total knee arthroplasty is decreasing, the survival of implants is becoming a more important factor in selection of fixation. The related literature and meta-analysis revealed that the uncemented knee arthroplasty has comparable survival rate as cemented prostheses in short term follow up, and uncemented arthroplasty is suggested to young and obese patients. However, ideal fixation method is still debated, and most of the surgeons prefer cemented knee arthroplasty.

Key words: primary total knee arthroplasty; cemented; cementless; outcomes; aseptic loosening

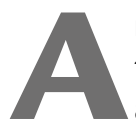

rtroplasti kayıtlarına göre, altmış yaş altı hastalarda primer diz protezi uygulanması hızla artmaktadır. ${ }^{[1,2]}$ Bunun sonucu olarak revizyon olguları da artmakta, 2030 yılında, revizyon sayısının tüm total diz protezi (TDP) uygulamalarının \%14,5'ine ulaşacağı öngörülmektedir. ${ }^{[2]}$ TDP'de ana amaç, ağrısız, fonksiyonel, hastanın beklentilerini karşılayacak ve en az $15-20$ yıllık sağkalım oranı $\% 90$ olan bir diz elde etmektir. ${ }^{[3]}$ Her ne kadar primer TDP'de 10 yıllık sağkalım \%90'ın üzerinde olsa da, 75 yaş üstü hastalarla karşılaştırıldığında, 65 yaş altı hastalarda revizyon oranı 2,5 kat, 55 yaş altı hastalarda ise beş kat daha fazladır. ${ }^{[4]}$ TDP'nin sağkalım süresini, temel olarak hastanın aktivite düzeyi, yaşı, kemik kalitesi gibi hastaya bağlı faktörler belirler. Bunun yanı sıra, implantın tasarımı ve tespit türü de sağkalım üzerine etkilidir. ${ }^{[5]}$ Aseptik gevşeme, revizyon olgularının \%40'ını oluşturur ve en sık revizyon nedenidir. ${ }^{[6]}$ Ancak, TDP gün geçtikçe daha genç, aktif ve obez hastalarda uygulanmaktadır. ${ }^{[1,7-9]} \mathrm{Bu}$ da, komponentlerde gevşeme ve revizyon ihtimalini arttırmaktadır. TDP'de ideal komponent tespit metodu halen tartışmalıdır. Çimentolu

- İletişim adresi: Dr. Ömer Faruk Bilgen, Özel Medicabil Hastanesi, Fethiye Mah. Küre Sokak No:1 Nilüfer, Bursa, Türkiye Tel: 0224 - 2415533 e-posta: ofbilgen@gmail.com

- Geliș tarihi: 24 Ocak $2019 \quad$ Kabul tarihi: 24 Ocak 2019 


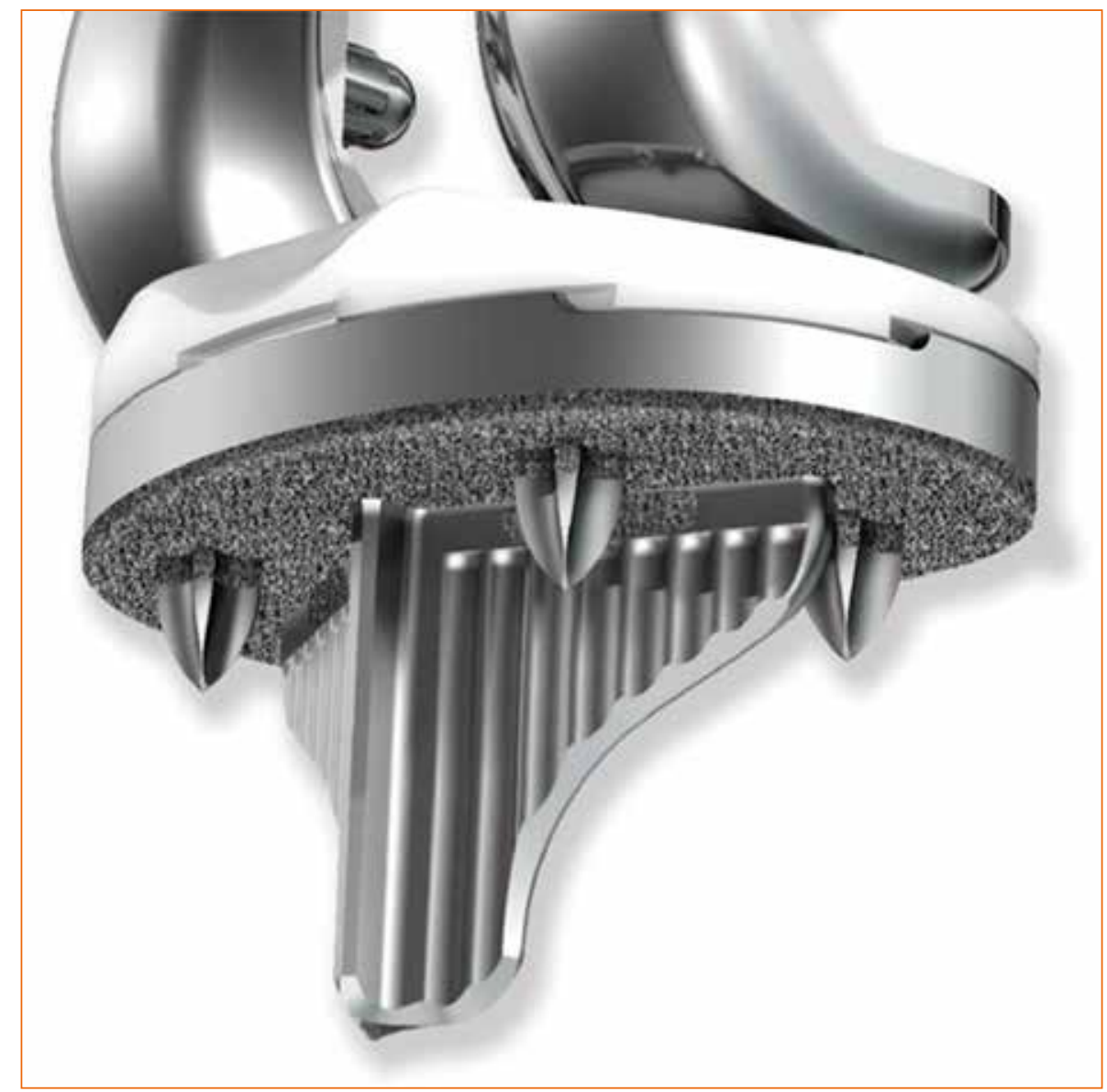

Şekil 1. Poroz yüzeyli çimentosuz tibial komponent (Stryker $\left.{ }^{\circledR}\right)$.

TDP ile mükemmel klinik sonuçlar elde edilmiş, uzun dönem takiplerde düşük oranlarda aseptik gevşeme görülmüş ve bu, diz protezi uygulamalarında en çok kullanılan metod olmuştur. Ancak, kemik çimento ara yüzündeki değişiklikler osteolize neden olmakta, özellikle genç hastalarda bu komplikasyonu önlemek için yeni tespit yöntemleri aranmaktadır. ${ }^{[3]}$ Genç hastalarda çimentosuz diz protezi uygulamaları seksenli yılların ortalarında başlamış, ancak kısa ve orta dönem takiplerde yüksek oranda gevşeme görüldüğü için standart çimentolu protezlere geri dönülmüştür. Uygulanan ilk çimentosuz protezlerde problemler; protez tasarımında başarısızlık, yeterli ve devamlı poroz yüzeyin elde edilememesi, kemik protez tespitinin yetersiz olması, birinci nesil konvansiyonel polietilen kullanılması, metal arkalıklı patellar komponent kullanımı olarak sıralanabilir. ${ }^{[10]}$ Yükssek poroz kaplı yüzeyler geliştirilmesi, biyolojik tespite güç katmakta ve çimentosuz TDP'ye olan ilgiyi arttırmaktadır (Şekil 1).

\section{ÇIMENTOLU TOTAL DIZ PROTEZi}

Çimentolu tespit TDP'de altın standarttır. Yumuşak doku dengesinin sağlanması, kemik yüzeylerin hazırlanması, çimento uygulama ve özenli titiz cerrahi uzun ömürlü protez için gereklidir. Fakat protez-çimentokemik ara yüzleri arasında sağlam ve erken tespitin sağlanması, TDP'de mekanik gevşemenin önlenmesinde ve uzun dönemde iyi sonuçlar elde etmede en önemli etkendir. ${ }^{[4]}$ Çimentolu TDP, özellikle romatoid artrit gibi inflamatuvar hastalıklara bağlı, kemik kalitesi kötü hastalarda endikedir. Bunun yanı sıra, revizyon cerrahisinde, diyabetik hastalarda, immünsupresif hastalarda antibiyotikli çimentolar da kullanılabilir. Ayrıca, endikasyondan bağımsız olarak, birçok çalışmada çimentolu tespitle daha iyi sonuçlar elde edildiği bildirilmiştir. ${ }^{[11]}$

Birçok cerrah, ileri düzeyde kardiyopulmoner hastalığı olan kişilerde çimentolama sırasında oluşabilecek 
emboli riskinden dolayı çimentolu tespitten kaçınmaktadır. Ayrıca, kemik kalitesi iyi olan genç hastalarda da çimentolu tespit önermeyen cerrahlar vardır ama bu tercih meselesidir. Çimentolu tespit ile, uzun dönem takiplerde iyi-mükemmel sonuçlar bildirilmiştir. ${ }^{[12]}$

Çimentolu TDP'ye alternatif olarak çimentosuz tespitler kullanılır. Kemik kalitesi iyi olan genç hastalarda çimentosuz TDP uygulanabilir, ancak bu hasta grubunda dahi tibial komponentte kemik içine büyüme gerçekleşmeyebilir ve protezde çökme görülebilir. Öte yandan, femoral komponentin çimentosuz tespiti birçok hastada başarılı olmuştur. ${ }^{[1]]}$

Komponent tespitinin başarısı için çimentolama dikkatli yapılmalıdır. En önemli faktör, çimentonun uygun basınçla kuru kansellöz kemik yüzeye uygulanmasıdır. Eğer sklerotik yüzeyler varsa, subkondral kemiğe çimentonun penetre olması için matkapla delikler açılmalıdır. Protez ömrünün uzun olmasında diğer bir faktör ise, çimentonun kemik yüzeyden en az 2-3 mm derine penetre olmasıdır. Tibial komponentin anterior-posterior (AP) planda $3^{\circ}$ 'den fazla varus veya valgusta olması, asimetrik yüklenmeye ve erken gevşemeye neden olacaktır. Ayrıca, çimentonun polimerizasyonu sırasında komponentlerin hareket etmesinden kaçınmak gerekir. ${ }^{[6]}$

Sonuç olarak; çimentolu TDP ile uzun dönemde birçok implant türünde iyi-mükemmel sonuç elde edilmiştir (Tablo 1).

\section{ÇIMENTOSUZ TOTAL DIZ PROTEZI}

Çimentosuz TDP'nin başarılı ve uzun ömürlü olması için kemik içine büyümenin gerçekleşmesi gerekir. Bunun için de; implant kemik stabilitesi sağlam olmalı, kemik kalitesi ve kemik kan akımı iyi olmalıdır. Bu yüzden, çimentosuz TDP, kemik stoğu iyi olan ve vasküler sorunları olmayan hastalarda uygulanabilir. Bu hastalar, genelde 70 yaş altında ve yandaş medikal sorunları olmayan hastalardır. Hem erkek hem de kadın hastalarda çimentosuz TDP uygulanabilir; ancak, erkeklerde kemik dansitesi daha yüksek olduğundan, bu hastalarda endikasyon daha geniştir. ${ }^{[13]}$ Çimentosuz TDP, genelde primer osteoartrit, post-travmatik artrit ve iyi kontrol edilmiş romatoid artritte uygulanır. Her hasta ayrı ayrı değerlendirilmelidir. Kemik dansitesi iyi kemik kan akımı yeterli ise, yaş ve cinsiyetten bağımsız olarak her hastaya çimentosuz TDP uygulanabilir. ${ }^{[14]}$

Kemik stoğu yetersiz ve vasküler sorunu olan hastalarda çimentosuz TDP kontrendikedir. Diyabet, aterosklerotik vasküler hastalıklar ve dolaşımı bozan diğer hastalıklarda ise göreceli kontrendikedir. Aynı şekilde, sigara kullanımı da dolaşımı bozduğundan, göreceli kontrendikasyonlar arasındadır. Osteoporotik kemikte rijid tespit sağlamak mümkün olmadığı için, çimentosuz TDP osteoporoz hastalarında kontrendikedir. Bu durumda, implantta mikro-hareket olacak, o da implantın gevşemesine yol açacaktır. Aynı şekilde, ileri yaş hastalarda ve sedanter hayatı olan hastalarda $\mathrm{da}$, kemik stoğunun yetersiz olabileceği göz önüne alınarak, cerrahi sırasında çimentolu tespite dönme olasılığı göz ardı edilmemelidir. ${ }^{[11]}$

Çimentosuz TDP'nin, çimentolu TDP ile kıyaslanır düzeyde uzun dönem mükemmel sonuçları olabilir. Son çalışmalarda, 7-15 yıllık sağkalım \%87-100 arasında, 10 yıllık implant sağkalım ise $\% 95$ olarak bildirilmiştir (Tablo 2). Ancak, bu uzun dönem iyi sonuçların aksine, eski birçok çalışmada, özellikle tibial komponentte erken dönem gevşemeler bildirilmiştir. Bu çalışmalarda kullanılan implant tasarımları, artık modern çimentosuz implantlarda kullanılmamaktadır. Bazı erken tasarımlarda, tibial komponentte stem ve/veya vida kullanılmaması sonucu, ilk tespit yetersiz olmuş, kemik ile implant arasında fibrotik doku oluşmuş ve komponentte çökme meydana gelmiştir. ${ }^{\left[{ }^{[9]}\right.}$ Bir başka sorun ise tibial komponentin poroz kaplamasından kaynaklanmaktadır. Poroz kaplama çevresel olmadığında, polietilen debrisi düz yüzeyden kemiğe, oradan da vida deliklerine veya steme ilerlemekte, osteolize neden olmaktadır. Birçok eski çalışmada, ince-düz tibial polietilen kullanılmıştır. Bu, hem çimentolu hem de çimentosuz TDP'de erken dönem başarısızlık nedeni olmuştur. Ayrıca, metal arkalıklı ince polietilenli patellar komponent kullanımında metal-on-metal, abrazif aşınmaya neden olmuştur. ${ }^{[3]}$

Yeni implant tasarımı ve cerrahi tekniklerin gelişmesi ile birlikte, çimentosuz TDP uzun dönem sağkalım oranları çimentolu proteze eşit hale gelmiş, birçok çalışmada mükemmel sonuçlar bildirilmiştir (Tablo 1). Çimentolu TDP ile ilgili çalışmalarda, genç ve kilolu hastaların sonuçlarının daha yaşlı ve daha zayıf hastalara göre kötü olduğu gösterilmiştir. Ayrıca, çimentosuz TDP ile, genç ve kilolu hastalarda yaşlı ve zayıf hastalara benzer sonuçlar elde edilmiştir. Bu durumda, çimentosuz TDP genç ve obez hastalarda daha uygun bir seçenek olarak karşımıza çıkmaktadır. ${ }^{[15]}$

Çimentosuz TDP'de uygun hasta seçimi ve doğru cerrahi teknik, komplikasyonları ve başarısızlığı önlemede kritik önem taşır. Komponentlerin doğru dizilimi çok önemlidir. Böylece, kemik ve implant arasında kenar yüklenmeleri ve makaslama stresi önlenmiş olur. Bu makaslama gerilimi, özellikle tibial komponentte mikro-hareketlere yol açar, bu da kemik içine büyüme yerine fibrozis gelişmesine neden olur. Kemik kesilerin tam olması ve düz yüzey elde etmek önemlidir. Kesilerde yüksek bir noktanın olması kemik implant temasını azaltacak, kemik içe büyümesini sınırlayacaktır. Bunu önlemek, tam düz olmayan kesi yüzeyleri eşitlemek ve 
Tablo 1. Çimentolu TDP sonuçları

\begin{tabular}{|c|c|c|c|c|}
\hline Yazar & Diz sayısı & Ortalama yaş & Ortalama takip süresi & Sonuçlar \\
\hline Ranawat, et al. (1993) & 112 & 65 yaş & 15 yıl & \%94 iyi sonuç \\
\hline Gill and Joshi $(2001)^{[17]}$ & 268 & 68 yaş & $10,1 \mathrm{yll}$ & $\begin{array}{c}\text { Sağkalım } \\
10 \text { yılda \%98 } \\
17 \text { yılda \%92 }\end{array}$ \\
\hline Laskin $(2001)^{[18]}$ & 100 & 69 yaş & 10 yıl & Sağkalım \%97 \\
\hline Ritter $(2001)^{[19]}$ & 4583 & 70 yaş & 11 yıl & $\begin{array}{c}\text { Sağkalım } \\
15 \text { yılda } \% 98,86\end{array}$ \\
\hline Callaghan, et al. $(2005)^{[20]}$ & 119 & 70 yaş & $15-18$ yıl & Revizyonsuz \%94 \\
\hline
\end{tabular}

Tablo 2. Çimentosuz TDP sonuçları

\begin{tabular}{|c|c|c|c|c|}
\hline Yazar & Diz sayısı & Ortalama yaş & Ortalama takip süresi & Sonuçlar \\
\hline Berger, et al. $(2001)^{[14]}$ & 108 & 58 yaş & $11 \mathrm{yıl}$ & $\begin{array}{l}\text { Sağkalım } \\
\text { Femur \%88 } \\
\text { Tibia \%92 }\end{array}$ \\
\hline Whiteside $(2001)^{[21]}$ & 184 & 77 yaş & $15-18 \mathrm{yll}$ & $\begin{array}{l}\text { Sağkalım } \\
18 \text { yılda \%98,6 }\end{array}$ \\
\hline Goldberg $(2004)^{[22]}$ & 113 & 62 yaş & 14 yıl & Sağkalım \%87 \\
\hline Watanbe $(2004)^{[23]}$ & 50 & 72 yaş & 10 yıl & $\begin{array}{l}\text { Sağkalım } \\
10 \text { yılda } \% 100 \\
13 \text { yılda } \% 96,7\end{array}$ \\
\hline Hardeman $(2006)^{[24]}$ & 115 & 73 yaş & 10 yıl & Sağkalım \%97 \\
\hline $\operatorname{Baker}(2007)^{[13]}$ & 112 & 71 yaş & 10 yıl & Sağkalım \%93 \\
\hline
\end{tabular}

kemik içine büyümeyi arttırmak için, otolog kemikten elde edilecek sulu harç (bulamaç) kullanılabilir. ${ }^{[11]}$

\section{SONUÇLAR}

Finlandiya artroplasti kayıtlarına göre 1997-2003 tarihleri arasında 32.000 TDP yapılmış, bunların \%3'ü çimentosuz, \%6'sı ise hibrid sistemle uygulanmıştır. ${ }^{25]}$ Sibanda ve ark.'nın çalışmasına göre, Ingiltere'de yapılan TDP'lerin yaklaşık \%8'i çimentosuzdur. ${ }^{[26]}$ Çimentolu TDP'de üç yıllık revizyon oranı $\% 1,4$ çimentosuz TDP'de ise revizyon oranı \%1,5 bulunmuştur. 1997-2007 yılları arası çimentosuz TDP oranı (hibrid sistemler dahil) İsveç'te \%2, Norveç'te \%14, Danimarka'da ise \%22'dir. ${ }^{[27]}$

Pijls ve ark.'nın yaptıkları meta-analizde, Interax ${ }^{\circledR}$ komponentlerde migrasyonun fazla olduğu, buna bağI olarak aseptik gevşeme ve revizyon oranının arttığı gösterilmiştir. ${ }^{[28]}$
Carlsson ve ark. üç tip protezi karşılaştırmış (çimentolu, çimentosuz poroz komponent, çimentosuz poroz hidroksiapatit kaplı komponent), sonuç olarak beş yıllık takipte çimentolu tespitin daha stabil olduğunu ortaya koymuşlar; ayrıca, çimentosuz sistemlerde hidroksiapatit kaplamanın implant ile kemik arasındaki hareketi azalttığını göstermiş̧lerdir. ${ }^{[29]}$

Gandhi ve ark.'nın 2009 yılında yayımladıkları meta-analizde, çimentolu implantların sağkalımının daha iyi olduğu gösterilmiş, ancak klinik sonuçlar açısından fark bulunamamıştır. ${ }^{[30]}$

Mont ve ark. 37 çalışmayı incelemiş (2940 hasta, 3568 TDP), çimentolu ve çimentosuz diz protezlerini karşılaştırmış, 10 yıllık sağkalım çimentosuz TDP'de \%95,6 çimentolu TDP'de \%95,3 bulmuş, 20 yıllık takipte ise sırasıyla $\% 76$ ve $\% 71$ olarak tespit etmişlerdir. Ayrıca, çimentosuz TDP'de vidalı veya vidasız tespit arasında fark görmemişlerdir. ${ }^{[31]}$ 
Lass ve ark., 60 çimentosuz TDP ile tibiası çimentolu / femuru çimentosuz 60 hibrid sistemi değerlendirmişler, hastaları beş yıllık radyolojik sonuç, klinik sonuç ve implant sağkalımı açısından karşılaştırmışlardır. Her iki grupta da ikişer hastada tibial gevşeme nedeniyle revizyon yapılmış \%96'lık implant sağkalımı görülmüştür. Radyolojik değerlendirmede, çimentosuz grupta tibial komponent çevresinde daha az radyolüsen alan gözlenmiştir. Ortalama altı yıllık takip sonunda, klinik ve fonksiyonel sonuçlarda gruplar arası fark gözlenmemiştir. ${ }^{[32]}$

Beaupré ve ark.'nın ileriye dönük randomize çalışmalarında, 70 yaş altı hastalarda çimentosuz hidroksiapatit kaplı tibial komponentlerle çimentolu tibial komponentler karşılaştırılmıştır. Beş yıllık takipte; ağrı, fonksiyon, yaşam kalitesi ve ameliyat sonrası komplikasyon ve radyolojik skorlar arasında gruplar arasında fark görülmemiştir. ${ }^{[33]}$

Prudhon ve Verdier, 2007 yılında yayımladıkları çaIsşmada, aynı tasarıma sahip 100 çimentolu TDP ile 100 çimentosuz TDP karşılaştırmışlardır. Hastaların ortalama yaşı 73'tür ve \%81'i primer osteoartrit nedeniyle ameliyat edilmiştir. On bir yıl sonunda, çimentolu grupta sağkalım \%90,2, çimentosuz grupta ise $\% 95,4$ bulunmuş, çimentosuz grupta sağkalım daha iyi olmasına rağmen istatistiksel olarak anlamlı fark saptanmamıştır. ${ }^{[34]}$

İmplant yüzeyindeki hidroksiapatit gibi biyoaktif kaplamalar, kemik içe büyüme hızını arttırmaktadır. Hidroksiapatit kaplama, kemik ile implant arasında destek oluşturup hareketi ve harekete bağlı fibrotik doku oluşumunu engellemektedir. ${ }^{[35]}$ Poroz yüzey ve hidroksiapatit kaplama çimentosuz TDP maliyetlerini arttırmaktadır. Fiyatlar, firmalara ve ülkelere göre değişiklik gösterir. Örneğin; Norveç'te çimentosuz protezler çimentolu protezlere göre \%30-40 daha pahalıdır. Çimentosuz TDP'de çimento maliyeti olmayacaktır; ayrıca, ameliyat süresi kısaldığı için ameliyat maliyetleri de azalacaktır. Çimentosuz TDP'nin çimentoluya göre maliyet farkını hesaplamak için kapsamlı bir hesap yapılmadır. Kamath ve ark.'nın çalışmasında, Zimmer ${ }^{\circledR}$ çimentosuz protez çimentolu bağ kesen proteze göre $\$ 596$ daha pahalıdır; ancak çimento, çimento karıştırma aparatları ve ameliyat süresi hesaba katılınca, maliyet farkı sadece $\$ 150$ olarak bulunmuştur. ${ }^{[36]}$

\section{TARTIŞMA}

1980'lerde ilk uygulandığında, kısa dönemde yüksek gevşeme ve revizyon oranları nedeniyle çimentosuz TDP'den vazgeçilmiş, şu anda bile altın standart olarak kabul edilen çimentolu TDP'ye dönülmüştür. Daha sonraları, yüksek poroz kaplamaların ve polietilenlerin iyileşmesi, hidroksiapatit gibi biyo-aktif tabakaların eklenmesiyle, çimentosuz TDP'ye olan ilgi tekrar artmış ve yapılan son çalışmalarda uzun dönem sağkalımın çimentolu ile karşılaştırılacak düzeye geldiği görülmüştür. Kim ve ark.'nın çalışmalarında, bilateral çimentolu ve çimentosuz TDP arasında, klinik, fonksiyonel ve radyolojik parametreler kıyaslandığında, her iki grup arasında anlamlı fark olmadığı bulunmuştur. ${ }^{[37]}$ Yine LizaurUtrilla ve ark., sağkalım oranlarına bakılırsa çimentolu ve çimentosuz TDP arasında fark olmadığını göstermişlerdir. ${ }^{[38]}$ Klinik uygulamalarda, genç aktif hastalara TDP uygularken, fonksiyonel ve uzun ömürlü bir diz için implantın tipi, tasarımı ve tespit türüne dikkat etmek gerekir. Genç hastalarda en önemli faktör, protezin sağkalım süresidir. Revizyon cerrahisinden sonra kemik kaybı göz önüne alınarak, daha iyi kemik stoğu sağlayacak implant ve tespit türü tercih edilmelidir. Çimentolu diz protezlerinde, revizyon cerrahisi sırasında çimentosuz proteze kıyasla daha fazla kemik rezeksiyonu gerektiği, klasik literatür bilgisidir. ${ }^{[39]}$ Ancak son yayınlarda, kemik entegrasyonu yüksek olan trabeküler metal yüzeylerde de revizyon sırasında kemik kaybının fazla olduğu bildirilmiştir. Elimizdeki bilgiler çimentolu sistemlerde kemik kaybının daha fazla olduğunu söylese de, çimentosuz sistemlerde de kemik kaybı olacağı göz ardı edilmemelidir. ${ }^{[3]}$

Eski çimentosuz protezlerdeki başarısız sonuçlardan sonra, birçok cerrah çimentolu tespiti tercih etmiştir. Değişik tip çimentolu protezlerde 10 yıllık sağkalım \%90'ın üzerinde bulunmuştur. Ancak, ulusal kayıtlara göre genç hastalarda revizyon oranı anlamlı olarak artmıştır. ${ }^{[40]}$ Çimentonun makaslama ve gerilme kuvvetlerine direnci düşüktür; bunun da zamanla çimento kemik ve/veya implant çimento ara yüzlerinin bozulmasına ve gevşemelere neden olacağı bilinmektedir. ${ }^{[41]}$ Çimentosuz diz protezinin teorik olarak birçok avantajı bulunmaktadır: kemik stoğunun korunması, kısa ameliyat süresi, çimento gibi üçüncü cisimlere bağlı aşınmanın önlenmesi, revizyonun kolay olması ve en önemlisi, kemik içine büyüme ile biyolojik tespit sağlanarak daha uzun ömürlü diz protezi elde edilebilmesidir. ${ }^{[9,10,41]}$ Gioe ve ark., 5760 primer TDP'nin \%40'ında revizyon nedeninin aseptik gevşeme olduğunu bildirmişlerdir. ${ }^{[9]}$ Çimentolu TDP'de de genç ve kilolu hastalarda aseptik gevşeme en önemli başarısızlık nedenidir. Abdel ve ark., obez hastalarda dizilimi iyi olan TDP'den sonra aseptik gevşemenin arttığını göstermişlerdir. ${ }^{[8]}$ Yine benzer şekilde Bagsby ve ark., obez hastalarla obez olmayan hastaları karşılaştırmışlar, obezlerde çimentolu TDP'nin sağkalımını $\% 89$, obez olmayan hastalarda \%99 olarak bildirmişlerdir. ${ }^{[42]}$ Meehan ve ark., çimentolu TDP'nin birinci 
yıl sonunda aseptik gevşeme oranının, 55 yaş altı hastalarda 65 yaş üstü hastalara kıyasla 4,7 kat fazla olduğunu bulmuşlardır. ${ }^{[43]}$

Çimentosuz TDP'nin ilk tasarımlarında kötü sonuçlar, en sık metal arkalıklı patellar komponentlerden kaynaklanmıştır. ${ }^{[44]}$ Çimentosuz patellar komponentlerdeki bu yüksek başarısızlık oranı, ortopedik cerrahları çimentolu protezlere yönlendirmiştir. Ancak, çimentosuz femoral komponentin uzun dönem sağkalımının mükemmel olduğu birçok çalışmada bildirilmiştir. ${ }^{[3,8,32]}$ Yine, eski tip tasarımlarda tibial komponentte de yüksek başarısızlık olmuştur. Illk çimentosuz tibial komponentlerde, implant ve kemik arasında ilerleyen radyolüsen çizgiler görülmüş, bunun sonucu olarak aseptik gevşeme gelişmiştir.[15] Çimentosuz TDP'nin başarılı olması için implantın ilk stabilitesinin çok iyi olması gerekir. Ancak, eski tip tibial komponentlerde bu stabilite sağlanamadığı için, kemik içine büyüme gelişmemiş ve gevşeme görülmüştür. Yeni tasarımlarda yüksek poroz yüzey, hiproksiapatit kaplama ile biyolojik tespit sağlanmış, uzun dönemde çimentolu TDP ile kıyaslanabilir sağkalım elde edilmiştir. Tibial komponentin tespitinde vida kullanılmış, ancak bu vidalar zamanla kanal görevi görüp debrisin komponente ilerlemesine osteoliz ve gevşemeye yol açmıştır. ${ }^{[7,14]}$ Bunun yanı sıra, tibial komponent tespitinin vidalı veya vidasız olması arasında fark olmadığı birçok çalışmada bildirilmiştir. ${ }^{[45]}$ Gelişen teknolojiyle birlikte, yüksek poroz yüzeyler elde edilmesiyle, çimentosuz tibial komponentin ilk tespitinde vidaya gerek kalmamıştır. Nam ve ark., çimentolu ve çimentosuz TDP'yi karşılaştıran çaış̧malarında, kan kaybı ve hemoglobin değişikliği bakımından fark görmemişler, ancak çimentosuz protezde ameliyat süresinin kısaldığını bildirmişlerdir. ${ }^{[0]}$

Sonuç olarak; hala total diz protezlerinin çimentolu mu yoksa çimentosuz mu yapılması gerektiği tartışmalıdır. ilk çimentosuz protezlerde yaşanan başarısız sonuçlar, tüm dünyada yaygın olarak çimentolu protezlerin kullanılmasına neden olmuştur. Biyoteknolojiler ve biyomateryallerdeki gelişmeler sayesinde, osteokondüktif özelliği yüksek protezler geliştirilmiş, özellikle genç hastalarda, biyolojik tespit yapıp protez sağkalımını arttırmak için kullanılmaya başlanmıştır. Ancak, bu protezlerin kullanılmaya başlanması hala yeni sayılacağından, uzun dönem sonuçları hala belirsizdir. Son yayınlarda, çimentosuz TDP'de sağkalım süresinin çimentolu protezle kıyaslanacak düzeyde olduğu bildirilmiş, ancak bir metodun diğerine üstünlüğü henüz ispatlanamamıştır. TDP'ye olan ihtiyaç giderek artmakta ve giderek daha genç popülasyona TDP uygulanmaktadır. Çimentolu protez ile uzun dönemde gevşeme olacağından, özellikle genç ve obez hastalarda tekrar çimentosuz proteze yönelim başlamıştır. Kısa dönemli çalışmalar ve meta-analizlerde, çimentolu protezin sağkalım açısından üstünlügü gösterilememiştir. Teorik olarak; ameliyat süresinin kısa olması, turnike kullanmama, revizyonda kemik kaybının az olması çimentosuz protezlerde avantaj, maliyetinin yüksek olması ise dezavantajdır. Çimentosuz TDP'nin üstünlüğünü veya gerekliliğini göstermek için, çimentolu ve çimentosuz diz protezlerini klinik, radyolojik, fonksiyonel ve hasta memnuniyeti bakımından karşılaştıran iyi tasarlanmış, randomize, ileriye dönük, uzun takip süreli çalışmalara ihtiyaç vardır.

\section{KAYNAKLAR}

1. Kurtz SM, Lau E, Ong K, Zhao K, Kelly M, Bozic KJ. Future young patient demand for primary and revision joint replacement: national projections from 2010 to 2030. Clin Orthop Relat Res 2009;467(10):2606-12. Crossref

2. Kurtz S, Ong K, Lau E, Mowat F, Halpern M. Projections of primary and revision hip and knee arthroplasty in the United States from 2005 to 2030. J Bone Joint Surg Am 2007;89(4):780-5. Crossref

3. Aprato $A$, Risitano $S$, Sabatini L, Giachino M, Agati G, Massè A. Cementless total knee arthroplasty. Ann Transl Med 2016;4(7):129. Crossref

4. Carr AJ, Robertsson O, Graves S, Price AJ, Arden NK, Judge A, Beard DJ. Knee replacement. Lancet 2012;379(9823):133140. Crossref

5. Franceschetti E, Torre G, Palumbo A, Papalia R, Karlsson J, Ayeni OR, Franceschi F. No difference between cemented and cementless total knee arthroplasty in young patients: a review of the evidence. Knee Surg Sports Traumatol Arthrosc 2017;25(6):1749-56. Crossref

6. Sharkey PF, Hozack WJ, Rothman RH, Shastri S, Jacoby SM. Why are total knee arthroplasties failing today? Clin Orthop Relat Res 2002;404:7-13. Crossref

7. Dalury DF. Cementless total knee arthroplasty: current concepts review. Bone Joint J 2016;98-B(7):867-73. Crossref

8. Abdel MP, Bonadurer GF, Jennings MT, Hanssen AD. Increased aseptic tibial failures in patients with a BMI $\geq 35$ and well-aligned total knee arthroplasties. J Arthroplasty 2015;30(12):2181-4. Crossref

9. Brown TE, Harper BL, Bjorgul K. Comparison of cemented and uncemented fixation in total knee arthroplasty. Orthopedics 2013;36(5):380-7. Crossref

10. Cherian JJ, Banerjee S, Kapadia $\mathrm{BH}$, Jauregui JJ, Harwin SF, Mont MA. Cementless total knee arthroplasty: a review. J Knee Surg 2014;27(3):93-8. Crossref

11. Berry DJ, Lieberman JR, Azar FM, editors. Advanced Reconstruction Knee. Rosemont, II: American Academy of Orthopaedic Surgeons (AAOS); 2011.

12. Buechel FF Sr, Buechel FF Jr, Pappas MJ, Dalessio J. Twentyyear evaluation of the New Jersey LCS Rotating Platform Knee Replacement. J Knee Surg 2002;15(2):84-9.

13. Baker PN, Khaw FM, Kirk LMG, Esler CNA, Gregg PJ. A randomised controlled trial of cemented versus cementless press-fit condylar total knee replacement:15-year survival analysis. J Bone Joint Surg Br 2007;89-B(12):1608-14. Crossref

14. Berger RA, Lyon JH, Jacobs JJ, Barden RM, Berkson EM, Sheinkop MB, Rosenberg AG, Galante JO. Problems with cementless total knee arthroplasty at 11 years followup. Clin Orthop Relat Res 2001;392:196-207. Crossref 
15. Miller AJ, Stimac JD, Smith LS, Feher AW, Yakkanti MR, Malkani AL. Results of Cemented vs Cementless Primary Total Knee Arthroplasty Using the Same Implant Design. J Arthroplasty 2017;33(4):1089-93. Crossref

16. Ranawat CS, Flynn JW, Saddler S, Hansraj KK, Maynard, MJ. Long-term results of the total condylar knee arthroplasty. A 15-year survivorship study. Clin Orthop Relat Res 1993;(286):94-102. Crossref

17. Gill GS, Joshi AB. Long-term results of cemented, posterior cruciate ligament-retaining total knee arthroplasty in osteoarthritis. Am J Knee Surg 2001;14(4):209-14.

18. Laskin RS. The Genesis total knee prosthesis: a 10-year followup study. Clin Orthop Relat Res 2001;388:95-102. Crossref

19. Ritter MA, Berend ME, Meding JB, Keating EM, Faris PM, Crites BM. Long-term follow-up of anatomic graduated components posterior cruciate-retaining total knee replacement. Clin Orthop Relat Res 2001;388:51-7. Crossref

20. Callaghan JJ, O'Rourke MR, lossi MF, Liu SS, Goetz DD, Vittetoe DA, Sullivan PM, Johnston RC. Cemented rotatingplatform total knee replacement: a concise follow-up, at a minimum of fifteen years, of a previous report. J Bone Joint Surg Am 2005;87(9):1995-8. Crossref

21. Whiteside LA. Long-term follow-up of the bone-ingrowth Ortholoc knee system without a metal-backed patella. Clin Orthop Relat Res 2001;388:77-84. Crossref

22. Goldberg VM, Kraay M. The outcome of the cementless tibial component: a minimum 14-year clinical evaluation. Clin Orthop Relat Res 2004;428:214-20. Crossref

23. Watanabe H, Akizuki S, Takizawa T. Survival analysis of a cementless, cruciate-retaining total knee arthroplasty: clinical and radiographic assessment 10 to 13 years after surgery. J Bone Joint Surg Br 2004;86-B(6):824-9. Crossref

24. Hardeman F, Vandenneucker $H$, van Lauwe J, Bellemans J. Cementless total knee arthroplasty with Profix: a 8- to 10-year follow-up study. Knee 2006;13(6):419-21. Crossref

17. Julin J, Jämsen E, Puolakka T, Konttinen YT, Moilanen T. Younger age increases the risk of early prosthesis failure following primary total knee replacement for osteoarthritis: a follow-up study of 32, 019 total knee replacements in the Finnish Arthroplasty Register. Acta Orthop 2010;81(4):413-9. Crossref

18. Sibanda N, Copley LP, Lewsey JD, Borroff M, Gregg P, MacGregor AJ, vander Meulen JH. Revision rates after primary hip and knee replacement in England between 2003 and 2006. PLoS Med 2008;5(9):e179. Crossref

19. Robertsson O, Bizjajeva S, Fenstad AM, Furnes O, Lidgren L, Mehnert F, Odgaard A, Pedersen AB, Havelin LI. Knee arthroplasty in Denmark, Norway and Sweden: a pilot study from the Nordic Arthroplasty Register Association. Acta Orthop 2010;81(1):82-9. Crossref

20. Pijls BG, Nieuwenhuijse MJ, Schoones JW, Middeldorp S, Valstar ER, Nelissen RG. RSA prediction of high failure rate for the uncoated Interax TKA confirmed by meta-analysis. Acta Orthop 2012;83(2):142-7. Crossref

21. Carlsson $\AA$, Björkman A, Besjakov J, Önsten I. Cemented tibial component fixation performs better than cementless fixation: a randomized radiostereometric study comparing porous-coated, hydroxyapatite-coated and cemented tibial components over 5 years. Acta Orthop 2005;76(3):362-9. Crossref

22. Gandhi R, Tsvetkov D, Davey JR, Mahomed NN. Survival and clinical function of cemented and uncemented prostheses in total knee replacement: a meta-analysis. J Bone Joint Surg Br 2009;91-B(7):889-95. Crossref
23. Pivec R, Issa K, Kapadia BH, Maheshwari A, Harwin SF, Mont MA. Long-term implant survivorship of cementless total knee arthroplasty: a systematic review of the literature and metaanalysis. J Knee Surg 2014;27(5):369-76. Crossref

24. Lass R, Kubista B, Holinka J, Pfeiffer M, Schuller S, Stenicka $S$, Windhager R, Giurea A. Comparison of cementless and hybrid cemented total knee arthroplasty. Orthopedics 2013;36(4):e420-7. Crossref

25. Beaupré LA, Al-Yamani M, Huckell JR, Johnston DWC. Hydroxyapatite-coated tibial implants compared with cemented tibial fixation in primary total knee arthroplasty: a randomized trial of outcomes at five years. J Bone Joint Surg Am 2007;89(10):2204-11. Crossref

26. Prudhon JL, Verdier R. Cemented or cementless total knee arthroplasty?: Comparative results of 200 cases at a minimum follow-up of 11 years. SICOTJ 2017;3:70. Crossref

27. Søballe K, Overgaard S. The current status of hydroxyapatite coating of prostheses. J Bone Joint Surg Br 1996;78-B(5):68991. Crossref

28. Kamath AF, Lee GC, Sheth NP, Nelson CL, Garino JP, Israelite CL. Prospective results of uncemented tantalum monoblock tibia in total knee arthroplasty: minimum 5-year follow-up in patients younger than 55 years. J Arthroplasty 2011;26(8):1390-5. Crossref

29. Kim YH, ParkJW, Lim HM, Park ES. Cementless and cemented total knee arthroplasty in patients younger than fifty five years. Which is better? Int Orthop 2014;38(2):297-303. Crossref

30. Lizaur-Utrilla A, Miralles-Muñoz FA, Lopez-Prats FA. Similar survival between screw cementless and cemented tibial components in young patients with osteoarthritis. Knee Surg Sports Traumatol Arthrosc 2014;22(7):1585-90. Crossref

31. Corten K, MacDonald SJ. Revision TKA Component Removal. In: Bonnin M, Amendola A, Bellemans J, MacDonald S, Ménétrey J, editors. The Knee Joint, Surgical Techniques and Strategies. Paris: Springer-Verlag France; 2012. pp.1003-8.

32. Nam D, KopinskiJE, Meyer Z, Rames RD, Nunley RM, Barrack $\mathrm{RL}$. Perioperative and early postoperative comparison of a modern cemented and cementless total knee arthroplasty of the same design. J Arthroplasty 2017;32(7):2151-5. Crossref

33. Harwin SF, Elmallah RK, Jauregui JJ, Cherian JJ, Mont MA. Outcomes of a newer-generation cementless total knee arthroplasty design. Orthopedics 2015;38(10):620-4. Crossref

34. Bagsby DT, Issa K, Smith LS, Elmallah RK, Mast LE, Harwin SF, Mont MA, Bhimani SJ, Malkani AL. Cemented vs cementless total knee arthroplasty in morbidly obese patients. J Arthroplasty 2016;31(8):1727-31. Crossref

35. Meehan JP, Danielsen B, Kim SH, Jamali AA, White $\mathrm{RH}$. Younger age is associated with a higher risk of early periprosthetic joint infection and aseptic mechanical failure after total knee arthroplasty. J Bone Joint Surg Am 2014;96(7):529-35. Crossref

36. Ritter MA, Meneghini RM. Twenty-year survivorship of cementless anatomic graduated component total knee arthroplasty. J Arthroplasty 2010;25(4):507-13. Crossref

37. Ferguson RP, Friederichs MG, Hofmann AA. Comparison of screw and screwless fixation in cementless total knee arthroplasty. Orthopedics 2008;31(2):1-5. Crossref 